\title{
Meningioma in a Patient with Tall Cell Papillary Thyroid Carcinoma
}

Panagiotis Spyropoulos, Ifigenia Kostoglou-Athanassiou Endocrinologist, Athens, Greece Department of Endocrinology, Red Cross Hospital, Athens, Greece 


\section{Introduction}

Tall cell papillary thyroid carcinoma is known to be associated with an adverse outcome, namely with the early development of locally recurrent or metastatic disease

Therefore the administration of therapeutic radioactive iodine is considered absolutely necessary in the management of such patients

A meningioma may occur in a patient with another neoplastic disease, having also been described in patients with thyroid carcinoma 
Aim

The aim was to describe the case of a patient who presented with a tall cell thyroid carcinoma and was also found to harbor a meningioma 
A female patient, aged 59, presented with a thyroid nodule measuring $1.8 \mathrm{~cm}$ on ultrasound

Diagnostic evaluation involving thyroid hormone measurement, $99 \mathrm{mTc}$-scanning and fine needle aspiration biopsy revealed the presence of malignancy within a cold nodule

Surgery was performed

Histology revealed the presence of a tall cell thyroid carcinoma

Thereafter, the administration of a therapeutic dose of radioactive iodine was planned 


\section{Results}

A brain CT scan revealed the presence of a tumor in the area of the left temporal lobe

As tall cell papillary thyroid carcinoma may be associated with metastatic disease, the possibility of metastatic disease within the brain was discussed

In order to exclude the presence of tissue absorbing radioactive iodine in the brain a whole body scan with low dose 131! iodine was performed

The scan confirmed that the tumor was not absorbing radioiodine

Subsequently, a therapeutic dose of ${ }^{131}$ I was administered 


\section{Conclusions}

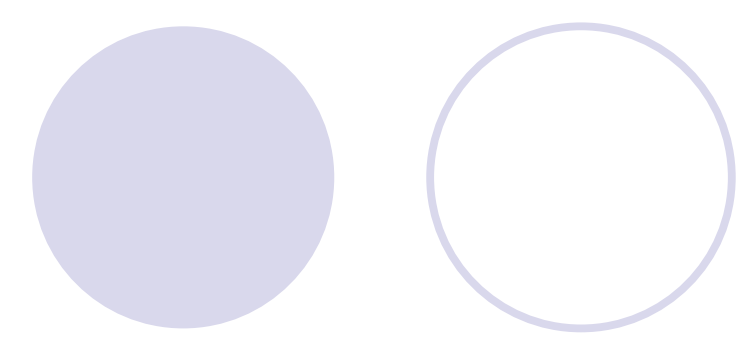

A meningioma may occur in a patient with another malignancy

In the case of a patient with thyroid cancer a meningioma can pose therapeutic difficulties, as it may mimic thyroid cancer metastatic disease in the brain, making specific diagnostic evaluation before the administration of therapeutic radioiodine necessary 